\title{
Simulating of OFDM Systems for Noise Abolition in the Long-Term Evolution application
}

\author{
${ }^{1 \text { st }}$ Mohammed Khaire Hussain, ${ }^{\text {2nd }}$ Firas Shawkat Hamid, ${ }^{\text {3rd }}$ Muhammed Khidr Jarjes \\ $\left\{\underline{\text { mohammed.khaire@ntu.edu.iq }}{ }^{1}, \underline{\text { dr.firas.hamid@ntu.edu.iq }}{ }^{2}\right.$ and $\left.\underline{\text { muhammedk.jarjes@ntu.edu.iq }}^{3}\right\}$
}

Mosul Technical Institute, Northern Technical University, +9647701812555',

Mosul Technical Institute, Northern Technical University, $+9647711199122^{2}$,

Technical College of Mosul, Northern Technical University, $+9647702026517^{3}$.

\begin{abstract}
Communication systems are basic in the modern world. OFDM has been used for noise abolition in LTE generation. In this paper, a simulation of OFDM system has been used by MATLAB GUI. Four cases have been studied, simulated, and analyzed. According to the type of noise in the channel, we used these parameters as an effect on the system. The effect of three parameters has been used for each case. The parameters include the number of the subcarrier in OFDM system, the number of samples on each subcarrier and the signal to noise ratio. The simulation used in this paper according to the mathematical communication equations gives accurate and fast results.
\end{abstract}

Keywords: OFDM, BER, SNR, MIMO, GSM, LTE, Mobile communications.

\section{Introduction}

Communication systems were very similar in modulating a transmitted signal on a carrier in RF using FM and dividing the users using FDMA regarding the mobile system AMPS (Advanced Mobile) $[1,2,3]$. The most famous system was NMT (Nordic Mobile Telephone) in northern Europe in 1981 as the first mobile system. In the United States AMPS was the first commercial mobile system in 1983. TACS was developed in Britain in 1985 based on AMS.

All the above systems suffer from incompatibility in a different frequency range, different operating systems, and different processing. Another important factor was the low capacity of the system. The second generation was the multi-digital system. As a result of digital transmit of communication systems and the need for transmitting voice information, fax, short message, and data exchange on the internet. Second generation includes GSM, D-AMPS,CDMA and PDC.

The third generation called $2.5 \mathrm{G}$ or GPRS is a developed version of GSM by sending and receiving internet pockets on a mobile network. The speed around $171.2 \mathrm{kbps}$. EDGE or $2.75 \mathrm{G}$ is a developed version of GSM/GPRS with a transmission rate of $384 \mathrm{kbps}$ with 8 PSK. Some of CDMA 2000 and EDGE protocols can be classified as the third generation. The fourth-generation main object ( $4 \mathrm{G}$ or beyond $3 \mathrm{G}$ ) is to enhance the QoS (quality of service) and maintain a high speed for data transmission in addition to developing multimedia message service the video conversation, highresolution TV in any time and place $[4,5,6]$.

It is characterized with high spectral efficiency i.e. increases the number of users who are able to use specified frequency band in one area (measured in bit /sec/Hz/cell) and increases in network capacity i.e. the number of users in the cell and the data rate of $(100 \mathrm{Mbit} / \mathrm{sec})$ when the user is moving with high speed and $(1 \mathrm{Gbit} / \mathrm{sec})$ for the reasonable stable user. Ensuring hand off easily between different networks. Multimedia with very high resolution and reaching integrated system which depend on IP completely i.e. the network depends completely on the transmission of pockets only. The technique of baseband depends on OFDM, MIMO, and STC [7, 8, 9, 10, 11, 12].

OFDM $[13,14,15,16]$ is a special case of multi-carrier modulation MCM. This technique increases the transmitted signal ability against the frequency selective fading and narrowband interference. Figure 1 shows a comparison between single carrier modulation SCM \& MCM with regard to the frequency spectrum. OFDM parameters include the number of subcarriers NSC, guard times, symbol duration, subcarrier spacing, carrier modulation techniques, and error correction techniques used. 


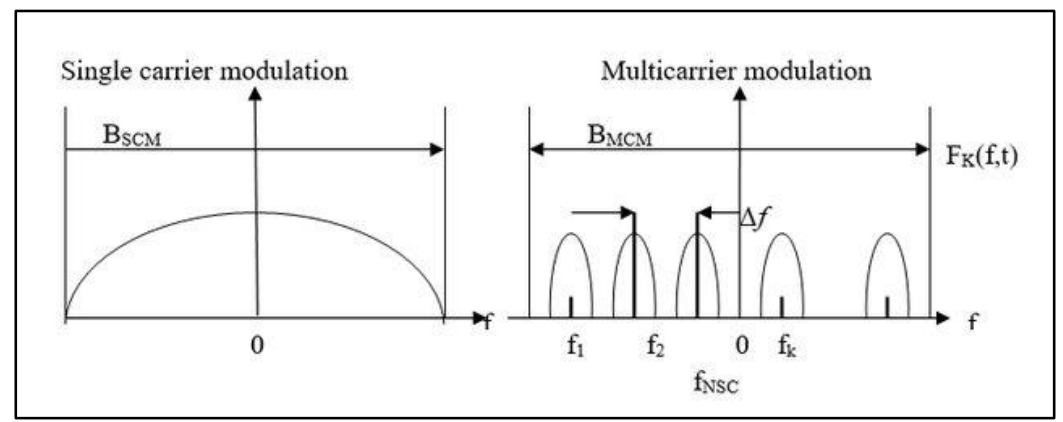

Fig. 1. Comparison of SCM and MCM

MIMO (multiple inputs multiple outputs) system consists of an array for transmission (MT) antennas and an array for reception (MR) antennas. For the Gaussian channel, the elements $\mathrm{S}$ will be independent identically distributed i.i.d. S represents the elements of transmission antennas and the transmission element from i antenna is $\mathrm{Si}[17,18,19,20,21,22,23]$.

\section{Theoretical part}

OFDM is regarded as an effective system in treating channels with Rayleigh fading with BER very close to non-faded signals. Effective use of bandwidth and no need for frequency spacing. solve the ISI with no need to use equalization in the receiver to solve the inter-carrier interference (ICI) problem.

The reduction in the signal to noise ratio because of the frequency drift is given by $[7,9,13]$ :

$$
\mathrm{D}=\frac{10}{3 \ln 10}\left(\prod \Delta \mathrm{ft}\right)^{2} \frac{\mathrm{Es}}{\mathrm{No}} \text {. }
$$

Where $\Delta \mathrm{f}=$ the frequency drift,

$\mathrm{T}=$ the inverse of subcarrier bandwidth,

$\frac{E s}{\text { No }}=$ The Signal to Noise Ratio.

In Figure 2 shows a decrease in SNR for three different values of SNR with three different modulation methods:
1.64 QAM $(\mathrm{Es} / \mathrm{No}=10 \mathrm{~dB})$
2. 16 QAM $(\mathrm{Es} / \mathrm{No}=14.5 \mathrm{~dB})$
3. QPSK $(\mathrm{Es} / \mathrm{No}=10.5 \mathrm{~dB})$

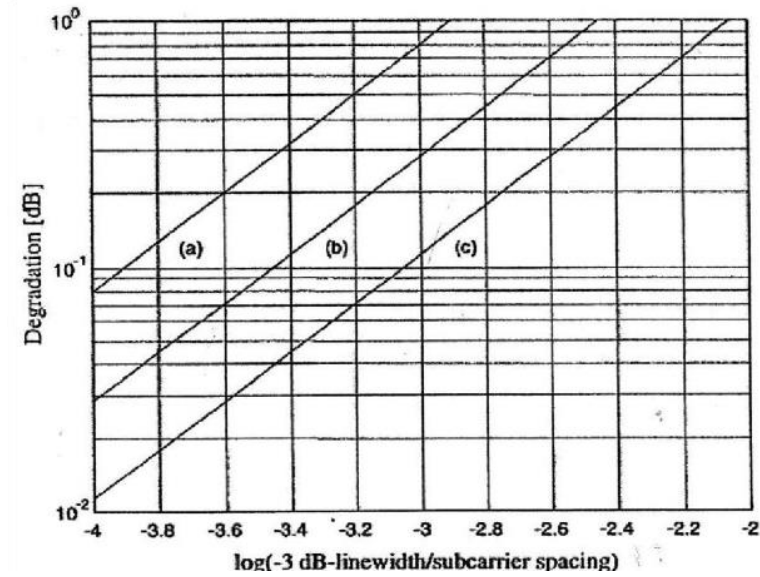

Fig. 2. Reduction of SNR following the frequency drifts for three modulation techniques.

There are three requirements for design of any system. The band width, the bit rate and the delay spread. 


\section{Simulation using MATLAB with Results}

MATLAB was used to design GUI that contains practical cases of OFDM system. This program has the ability to choose the number of carriers, SNR in addition to choosing the number of transmitted codes so one can make a comparison of results very easily.

\subsection{Case I}

In this case, the effect of white noise imposed on the signal is studied and the role of OFDM in cancelling this effect. Figure 3 below shows the case of OFDM with 64 QAM. The diagram consists of:

Data source: information signal is a random signal.

IQ MAPPER: QAM with 64 levels.

OFDM modulation: modulation of OFDM where samples are inputted \& carried by 16 subcarriers.

AWGN: where Gaussian noise is applied. IQ DEMAPPER: detection of QAM.

Data sink: here we get the output signal in $\mathrm{Rx}$

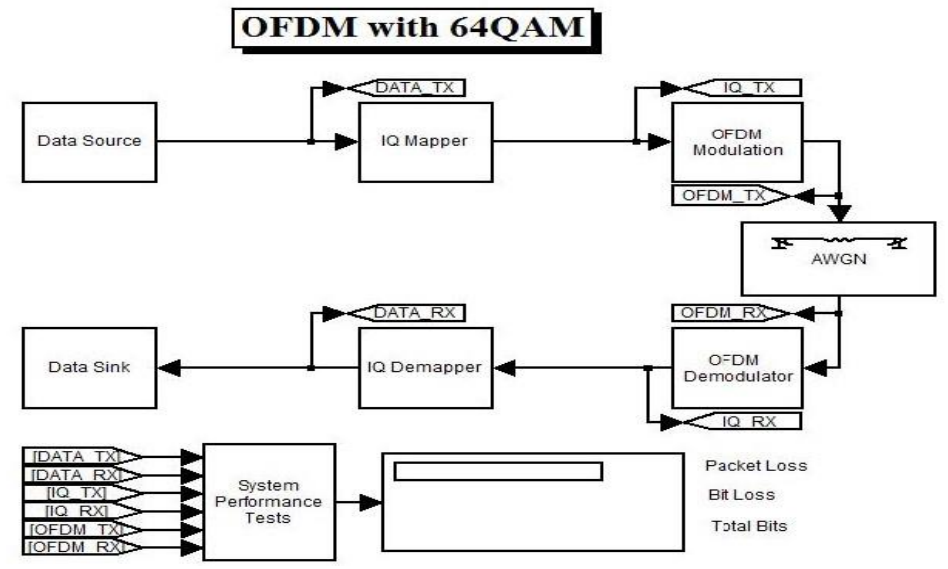

Fig. 3. Effect of WGN on the signal and the OFDM role in cancelling the effect

When this applied, we get 6 signals as shown in Figure 4 :

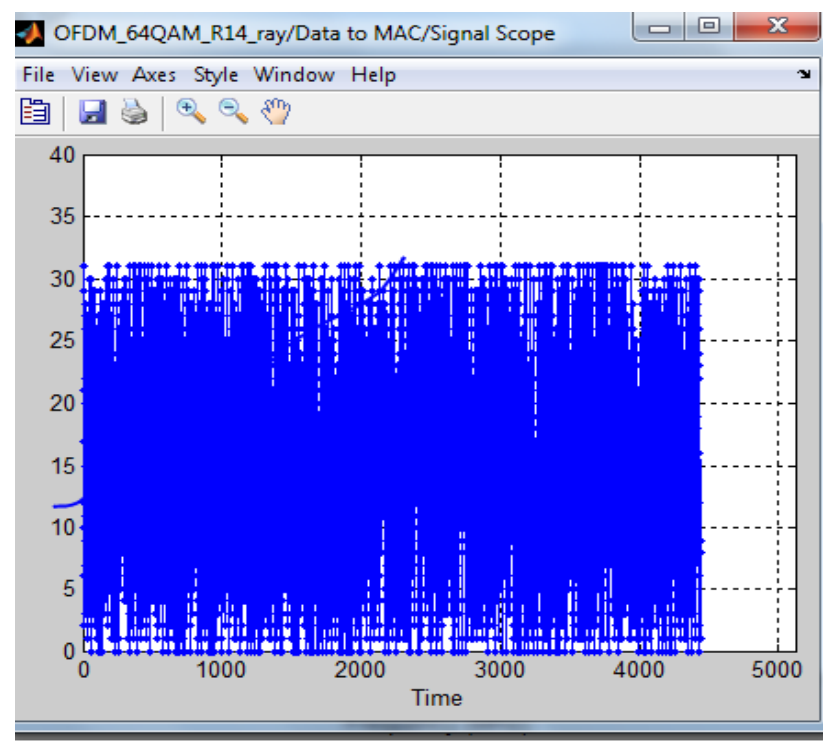

Fig. 4. input signal is a random signal.

It can be noticed that the signal is modulated on 16 subcarriers with different frequencies for each as shown in Figure 5 which shows QAM-64 signal. Figure 6 Shows OFDM signal 
modulated on 16 subcarriers for each a different frequency. Figure 7 shows OFDM signal with Gaussian noise.

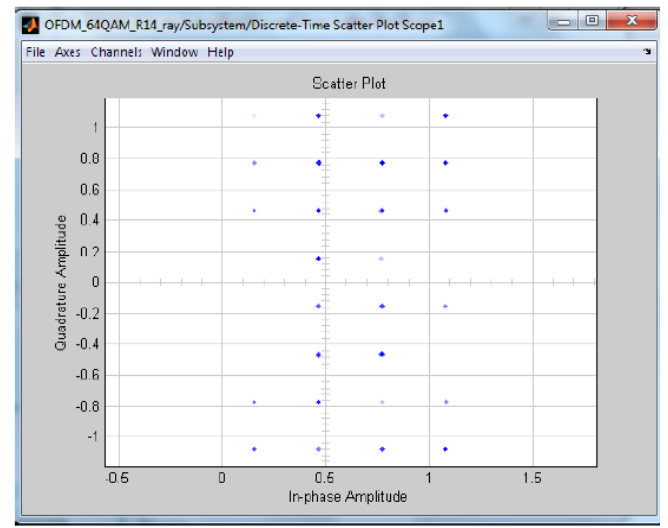

Fig. 5. QAM-64 signal

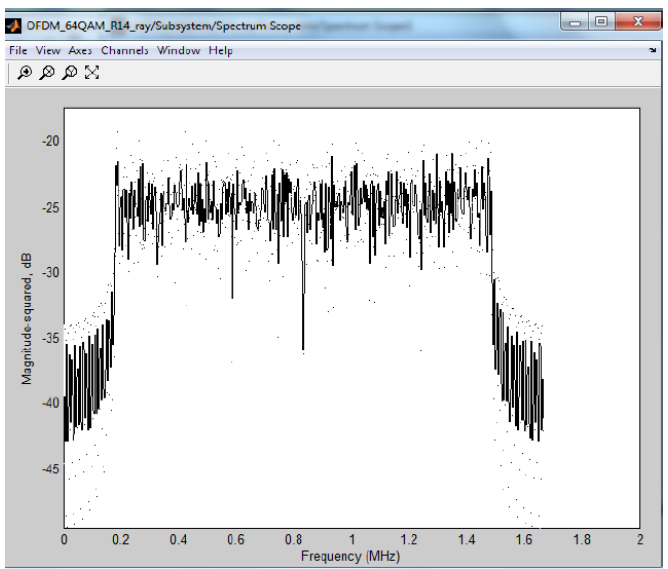

Fig. 7. OFDM signal with Gaussian noise

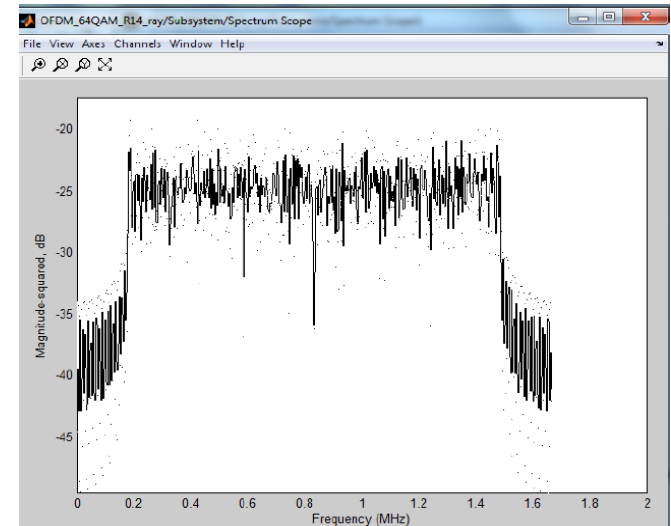

Fig. 6. OFDM signal is modulated on 16 subcarriers for each a different frequency

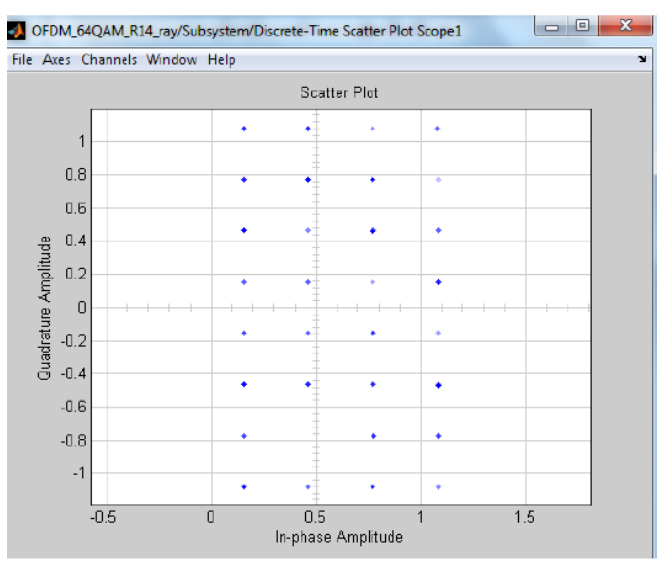

Fig. 8. The signal after the DEMAPPER

From Figure 6 and Figure 7 there is a small difference before and after adding the noise to the signal shown in Figure 8.

\subsection{Case II}

In this case, a study of the effect of Raleigh noise on the signal and the effect of OFDM system in removing it. Figure 9 shows a block diagram of OFDM with 64 QAM.

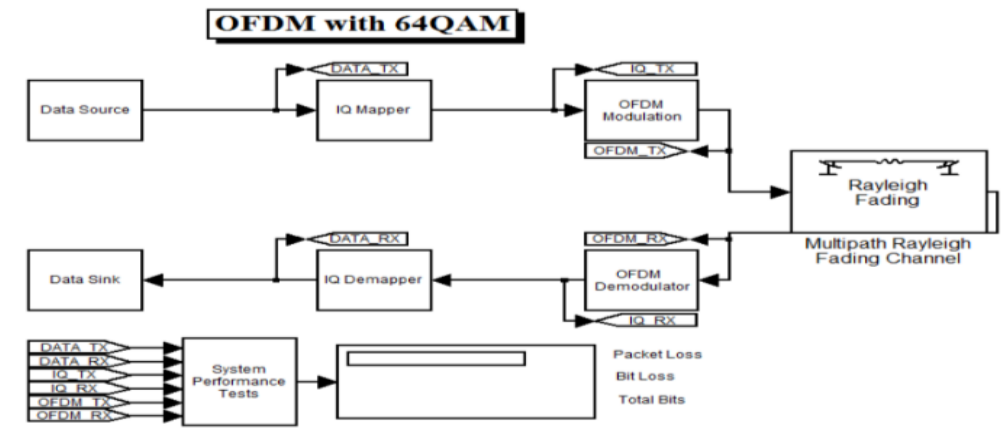

Fig. 9. Effect of Rayleigh fading on the signal and OFDM role in cancelling the effect. 
The diagram in Figure 9 differs from the previous diagram Figure 3 only with the type of noise in the channel. It is noticed that the random signal shown in figure 10 suffers from distortion at the end of the channel because of the Rayleigh effect as shown in Figure 11 and Figure 12.

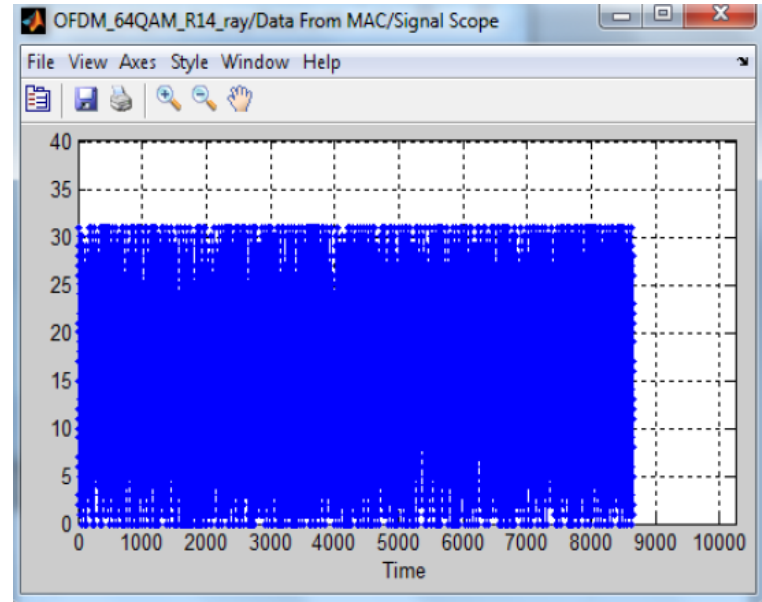

Fig.10. The input random signal.

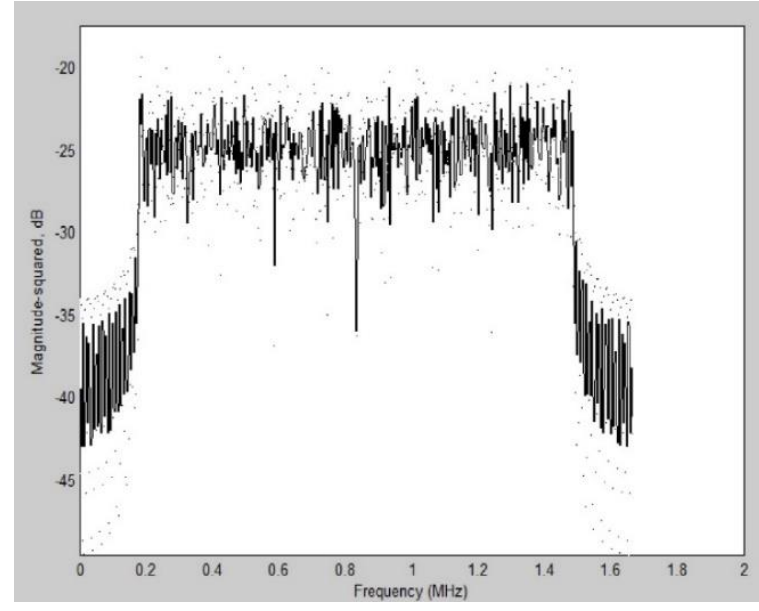

Fig.11. The signal before Rayleigh effect

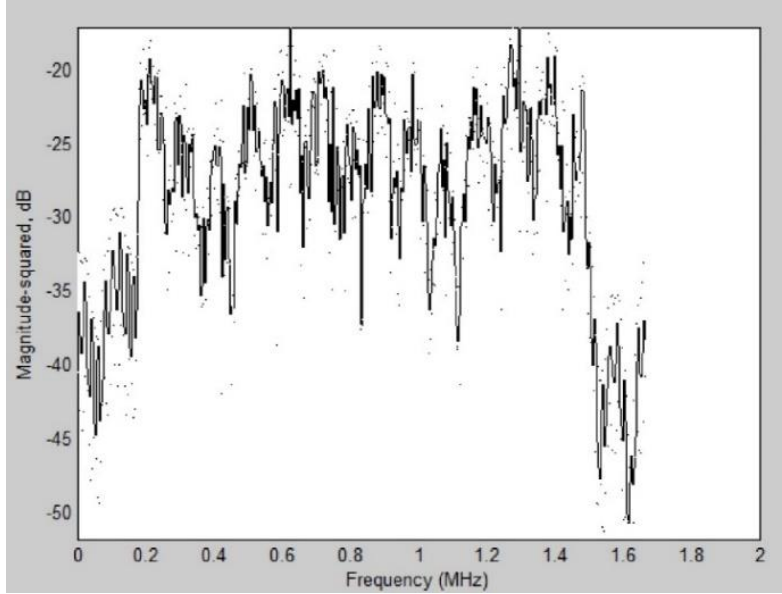

Fig.12. The signal after the Rayleigh effect.

\subsection{Case III}

In this case, the effect of three parameters on the system is studied:

1. Number of subcarriers in OFDM system.

2. The signal to noise ratio $\mathrm{Eb} / \mathrm{No}$.

3. Number of samples on each subcarrier.

\subsubsection{Case III A.}

The following parameters have been chosen for OFDM system (designed by Matlab) as shown in Figure 13 No. of sub carriers $=16, \mathrm{~Eb} / \mathrm{No}=0.510$, No. of symbols=20, with the 16 subcarriers as shown in Figure 14, Figure 15 shows that the theoretical signal is better than simulated signal, so the number of subcarriers is increased to 32 as shown in Figure 16. 


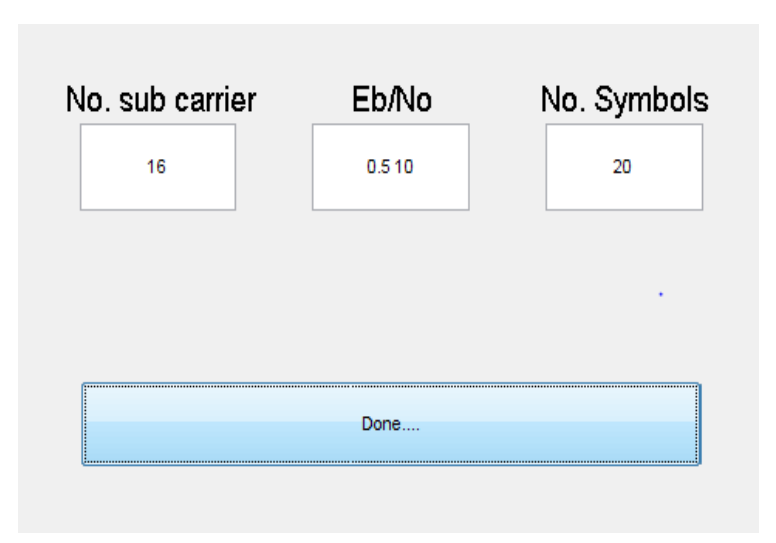

Fig.13. Control of system parameters with 16 subcarriers, 20 symbols

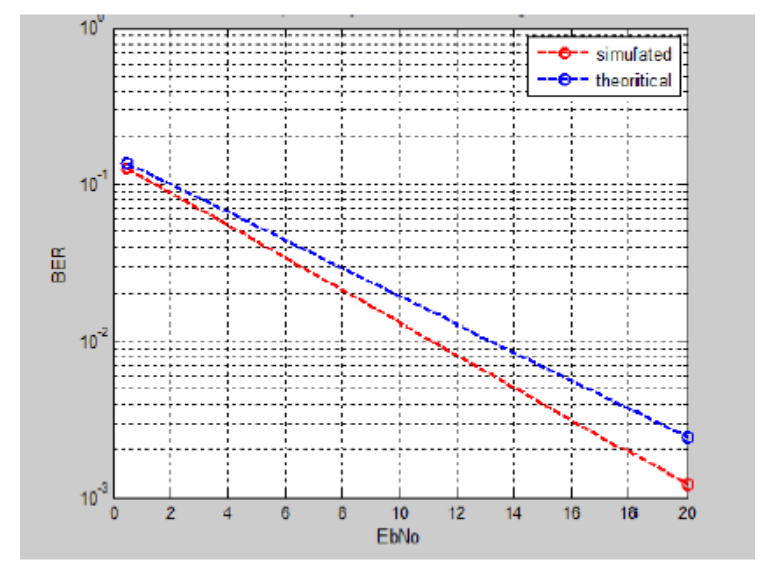

Fig.15. Comparison of theoretical and simulated 16 subcarriers OFDM.

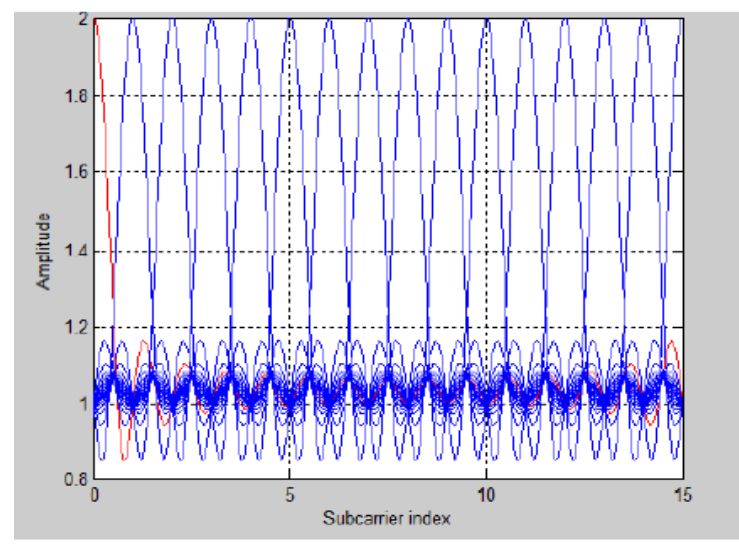

Fig.14. OFDM with 16 subcarriers

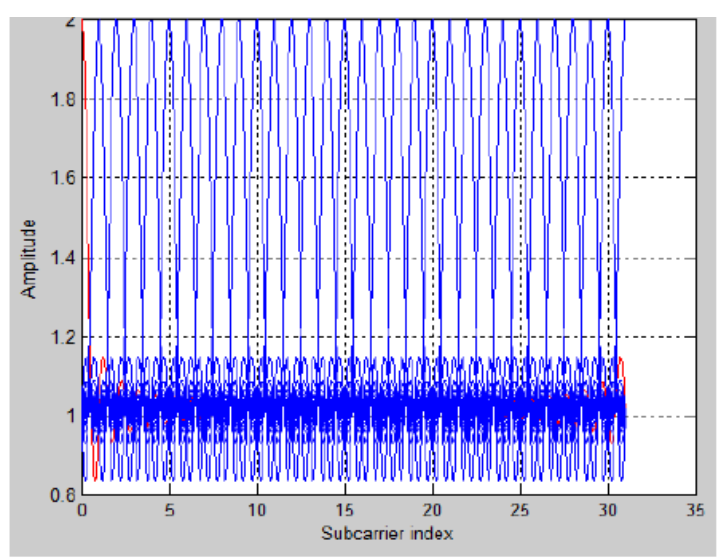

Fig.16. OFDM with 32 subcarriers.

Figure 17 shows better results because of the increase in the number of subcarriers to 64 as shown in Figure 18.

All these analyses show that an increase of subcarriers gives a better identical agreement of theoretical and simulated results, So the number of subcarriers increased to 64 as shown in Figure 18, almost identical agreement of theoretical and simulated results as shown In Figure 19.

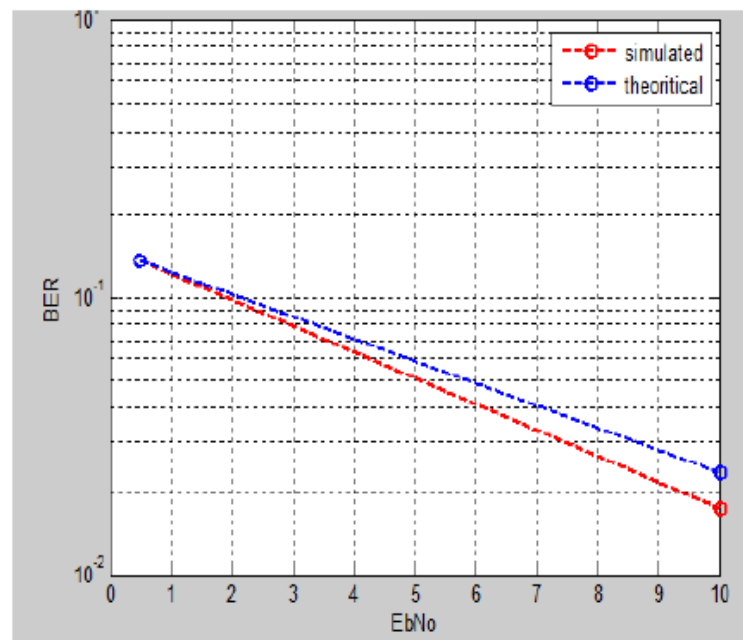

Fig. 17. A comparison of BER with SNR.

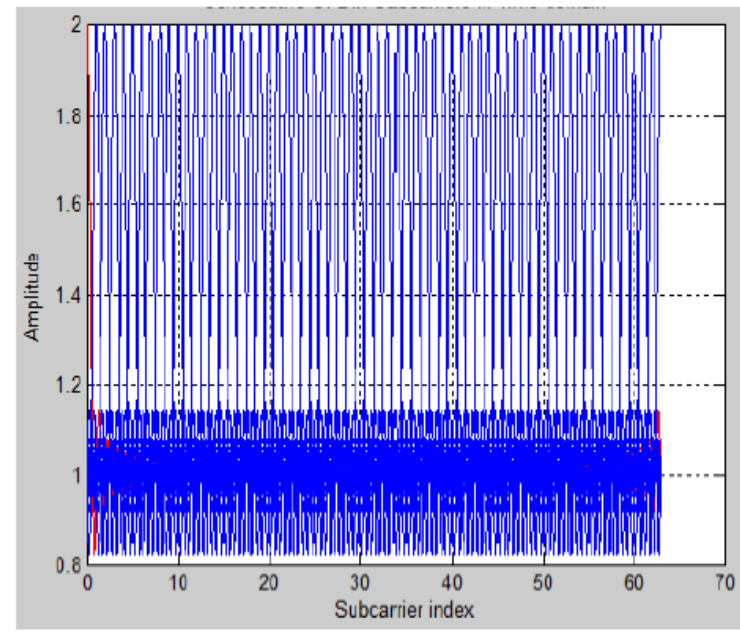

Fig. 18. OFDM with 64 sub carriers 


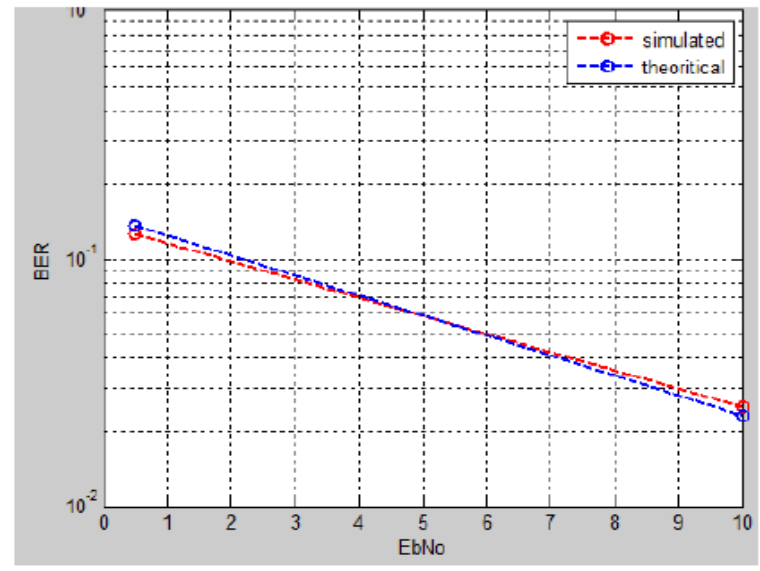

Fig. 19. A comparison of the theoretical and simulated BER and signal to noise ratio.

\subsubsection{Case III B.}

In this part, the signal to noise ratio is increased to show its effect as shown in Figure 20 that shows the effect of changing signal to noise ratio Eb/No in OFDM system when it has 16 subcarriers as shown in Figure 21.

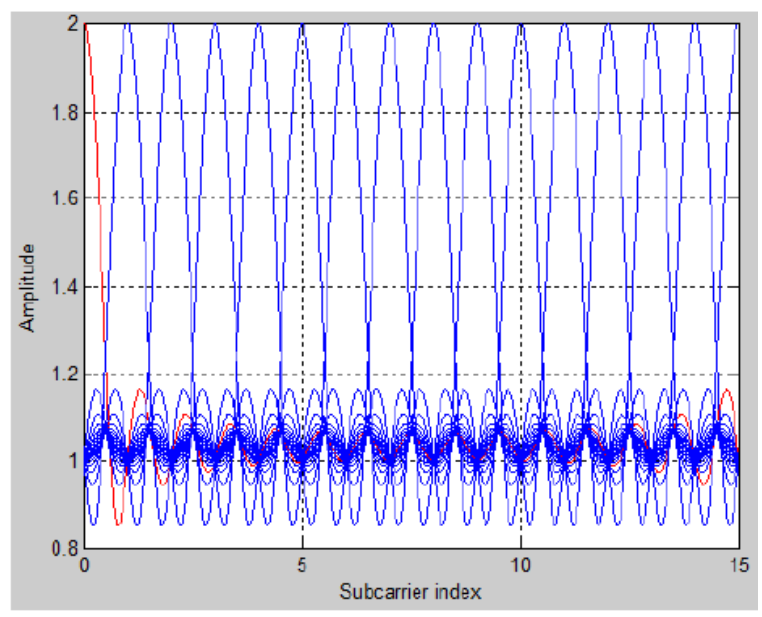

Fig.20: OFDM with 16 sub carriers

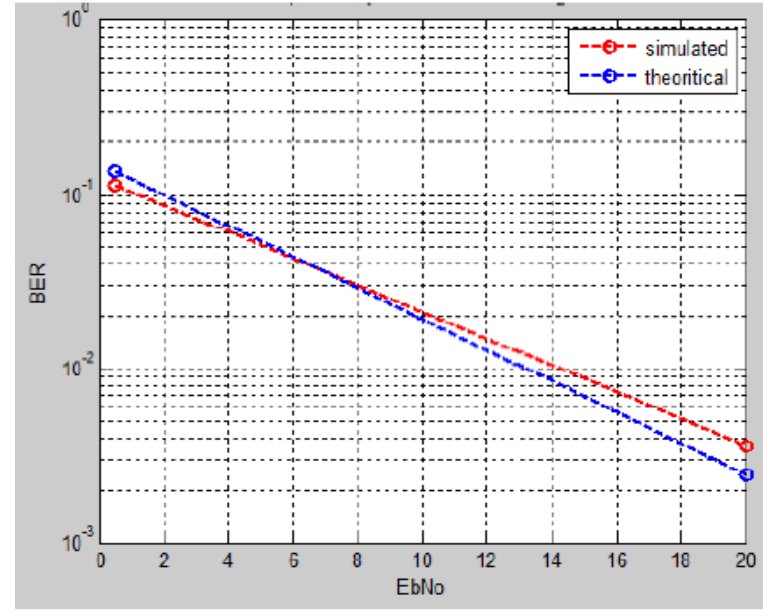

Fig.21: A comparison of the theoretical and simulated results of the signal to noise ratio and BER.

\subsection{Case IV}

\section{First application:}

In the first application the average bit error rate BER is drawn against the signal to noise ratio SNR for three different value of QAM with AWGN channel as shown in Figure 22.

Second application:

In the second application, the average bit error rate BER is drawn against the signal to noise ratio SNR for three different values of QAM with Rayleigh channel as shown in Figure 23.

\section{Third application:}

In the third application system the average BER against SNR for 4QAM system with different diversity order for Rayleigh channel as shown in Figure 24 :

\section{Fourth application:}

Diversity order $=2$, Diversity order $=4$.

In the fourth application as shown in Fig.26, the average bit error rate BER is drawn against the signal to noise ratio SNR for 4-QAM and 16-QAM for different diversity order as shown in Figure 25 :

4-QAM diversity order $=2,4$-QAM diversity order $=4$

16-QAM diversity order $=2,16-\mathrm{QAM}$ diversity order $=4$ 


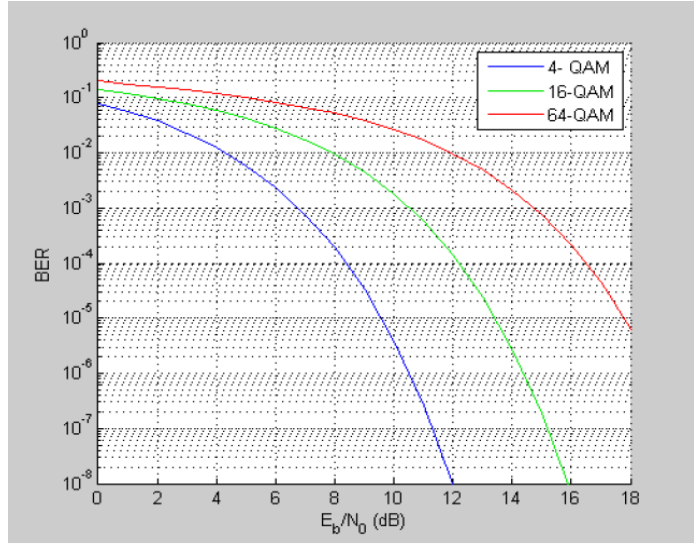

Fig. 22. BER vs SNR for three OFDM systems with AWGN channel

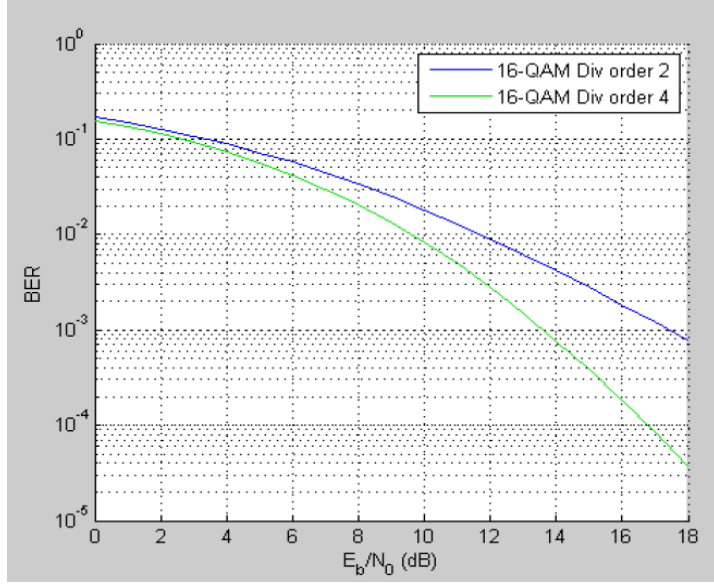

Fig. 24. BER vs SNR for two diversity order in Rayleigh channel.

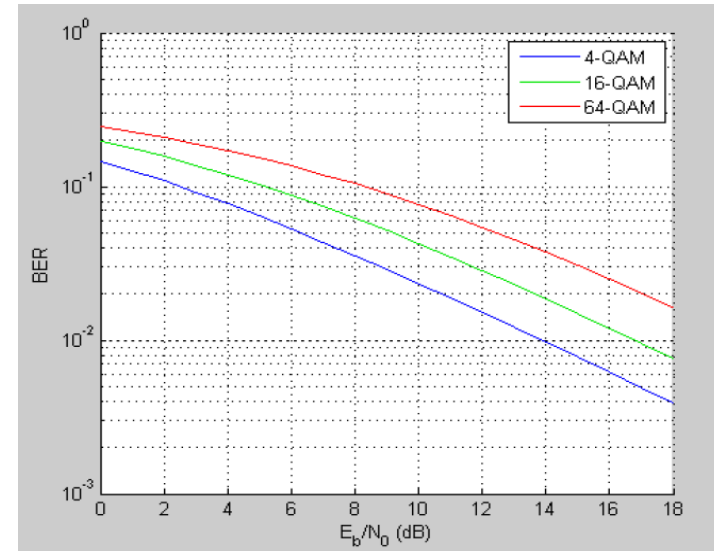

Fig.23. BER vs SNR for three OFDM systems with Rayleigh channel

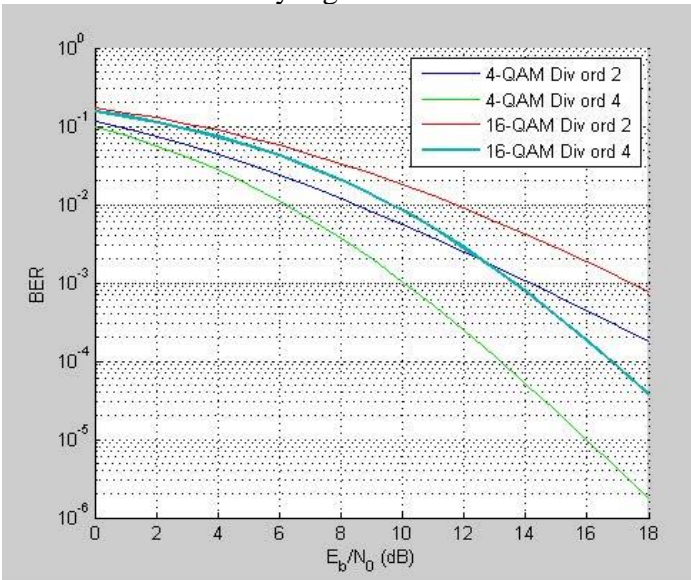

Fig. 25. BER vs SNR for three OFDM systems with Rayleigh channel for different diversity order.

\section{CONCLUSIONS}

In this paper, four simulated case studies have been performed using MATLAB that enables us to have accurate results to compare communication systems loaded with noise. Gaussian and Rayleigh noise distributions were used to represent the channel. From the results given in the practical part that include the number of subcarriers, the number of samples on each subcarrier, the signal to noise ratio and their effect on the system were analyzed. A Comparison of theoretical and practical results were addressed. For the fourth case study, the BER concerning the SNR for different channels and different diversity orders were also ilustrated.

\section{References}

[1] Zhancang, W.: High-Efficiency Load Modulation Power Amplifiers for Wireless Communications. Artech House (2017)

[2] Shinsuke, H. and Ramjee, P.: Multicarrier Techniques for 4GMobile Communication. Artech House (2003)

[3] Y., Li, F. Baccelli, J. G. Andrews, T. D. Novlan, and J. C. Zhang, "Modeling and analyzing the coexistence of wi-fi and LTE in unlicensed spectrum," CoRR, vol. abs/1510.01392, 2015. [Online]. Available: http://arxiv.org/abs/1510.01392.

[4] VAN, N. and Ramjee, P.: OFDM for Wireless Multimedia Communication. Artech House (2000)

[5] Peiwen, J. And Tianqi, W. and Bin, H., Xuanxuan, G., Jing, Z., .. and others, “Artificial Intelligenceaided OFDM Receiver: Design and Experimental Results", arXiv:1812.06638v2 [eess.SP] 20 Dec (2018) 
[6] Gao, X., Z, Huang., X. Lu, S. Zhang, C.-K. Wen, and S. Jin, "Implementation of massive MIMO uplink receiver on RaPro prototyping platform," IEEE Access, vol. 6, pp. 8133-8147, (2018)

[7] Hsiao-Hua C. and Mohsen G.: Next Generation Wireless System and Networks. Wiley and Sons (2006).

[8] Igor, R. Maravić and Aleksandar M. Nešković, "LTE Network Radio Planning”, Telfor Journal Vol.4, No.1, (2012)

[9] Ramjee, P.: OFDM for Wireless Communication System. Artech House (2004)

[10] [Henrik S. and Christian L.: Theory and Application of OFDM. John Wiley and Sons (2005)

[11] James, D. Gadze, Kwame A. Agyekum, Stephen J. Nuagah and E.A. Affum, "IMPROVED PROPAGATION MODELS FOR LTE PATH LOSS PREDICTION IN URBAN \& SUBURBAN GHANA", International Journal of Wireless \& Mobile Networks (IJWMN) Vol. 11, No. 6 , December (2019)

[12] Firas, S.: The difference between IEEE 802.16 / WiMAX and IEEE 802.11 / Wi-Fi networks for Telemedicine Applications. ISSN: 2277-3878, Vol. 2, Issue. 5. International Journal of Recent Technology and Engineering (IJRTE) (2013)

[13] Cisco, V. N. I. (2018). Cisco visual networking index: Forecast and trends, White Paper, 1. (2017)

[14] Tchao, E. T., Gadze, J. D., \& Agyapong, J. O. Performance evaluation of a deployed 4G LTE network. arXiv preprint arXiv:1804.05771. (2018)

[15] Kshirsagar, D. And Jain, S. And Sharma, S.: Review on multiple input multiple output antenna systems design. DOI: 10.1109/ICMOCE.2015.7489780. IEEE Xplore (2016)

[16] Firas, S.: GSM Modeling and Simulation for Urban Area. ISSN: 18131662. Vol. 15 Issue. 1, pp.126-136 Tikrit Journal of Pure Science (2010)

[17] Anand, M. Baswade, Mohith Reddy, Antony Franklin A, and Bheemarjuna Reddy Tamma,“ Modeling and Performance Analysis of Spatially Distributed LTE-U and Wi-Fi Networks", arXiv:1907.08954v1 [cs.NI] 21 Jul (2019)

[18] Gurudutt, H. Dandan, W. and Anil, R. "System Design Considerations For Internet Of Things (IoT) With Category-M Devices In LTE Networks", arXiv:1902.00408v1 [cs.NI] 1 Feb (2019)

[19] Selahattin G"okceli, Toni Levanen, Juha Yli-Kaakinen, Taneli Riihonen, Markku Renfors, and Mikko Valkama, "PAPR Reduction with Mixed-Numerology OFDM", arXiv:1909.02411v1 [eess.SP] 5 Sep (2019)

[20] T. Levanen, J. Pirskanen, K. Pajukoski, M. Renfors, and M. Valkama, "Transparent Tx and Rx waveform processing for $5 \mathrm{G}$ new radio mobile communications," IEEE Wireless Commun., vol. 26, no. 1, pp. 128-136, Feb. (2018)

[21] T. Shankar, A. Karthikeyan, Christopher Clement J, "Joint Estimation of Impairments for OFDM and MIMO OFDM Systems", International Journal of Computer Applications (0975 - 8887) Volume 23- No.5, June (2011)

[22] Yongli An, Liping Liu, Yan Liu and Yang Xiao,“ Effcient High Throughput Algorithm of MIMO LTE Network", Applied Mechanics and Materials, ISSN: 1662-7482, Vol. 443, pp 513-516 Trans Tech Publications, Switzerland (2014)

[23] Apostolis Galanopoulos and Antonios Argyriou, "Adaptive Video Streaming over LTE Unlicensed”, IEEE TRANSACTIONS ON MULTIMEDIA, arXiv:1608.00239v1 [cs.NI] $31 \mathrm{Jul}$ (2016). 